\title{
Simple Model for Encoding Natural Images by Retinal Ganglion Cells with Nonlinear Spatial Integration
}

\author{
Short title: Simple Model for Nonlinear Spatial Integration
}

\author{
Jian K. Liu ${ }^{1,2,3}$, Tim Gollisch ${ }^{1,2,4^{*}}$ \\ ${ }^{1}$ University Medical Center Göttingen, Department of Ophthalmology, 37073 Göttingen, \\ Germany \\ ${ }^{2}$ Bernstein Center for Computational Neuroscience Göttingen, 37073 Göttingen, Germany \\ ${ }^{3}$ School of Computing, University of Leeds, Leeds, LS2 9JT, UK \\ ${ }^{4}$ Cluster of Excellence "Multiscale Bioimaging: from Molecular Machines to Networks of \\ Excitable Cells" (MBExC), University of Göttingen, 37075 Göttingen, Germany \\ *Corresponding author, e-mail: tim.gollisch@med.uni-goettingen.de (TG)
}

\section{Abstract}

A central goal in sensory neuroscience is to understand the neuronal signal processing involved in the encoding of natural stimuli. A critical step towards this goal is the development of successful computational models of this encoding. For ganglion cells in the vertebrate retina, the development of satisfactory models for responses to natural visual scenes is an ongoing challenge. Standard models typically apply linear integration of visual stimuli over space, yet many ganglion cells are known to show nonlinear spatial integration in natural stimulus contexts. We here study the encoding of natural images by retinal ganglion cells, using multielectrode-array recordings from isolated salamander retinas. We assess how responses to natural and blurred images depend on first- and second-order statistics of spatial patterns inside the receptive field. This leads us to a simple extension of current standard ganglion cell models, which are based on linear spatial integration. We show that taking not only the weighted average of light intensity inside the receptive field into account but also its variance over space yields substantially improved response predictions of responses to novel images. Finally, we demonstrate how this model framework can be used to assess the spatial scale of nonlinear spatial integration. Our results underscore the importance of nonlinear spatial stimulus integration in the retina in responses to natural images. Furthermore, the introduced model framework provides a simple, yet powerful extension of standard models and may serve as a benchmark for the development of more detailed models of the nonlinear structure of receptive fields. 


\section{Author Summary}

For understanding how sensory systems operate in the natural environment, an important goal is to develop models that capture neuronal responses to natural stimuli. For retinal ganglion cells, which connect the eye to the brain, current standard models often fail to capture responses to natural visual scenes. This shortcoming is at least partly rooted in the fact that ganglion cells may combine visual signals over space in a nonlinear fashion. We here show that a simple model, which not only considers the average light intensity inside a cell's receptive field but also the spatial variance of the image, can partly account for these nonlinearities and thereby improve current standard models. This provides an easy-to-obtain benchmark for modeling ganglion cell responses to natural images.

\section{Introduction}

Much of our knowledge about how neurons in sensory systems operate stems from investigations with simplified, artificial sensory stimuli, whose properties can be specifically selected depending on the research question at hand [1]. Investigating the relevance of the inferred signal processing for real-life scenarios, however, requires examining responses of sensory neurons to natural stimuli [2-5]. An important step for this transition to natural stimuli is the design of appropriate computational models for the stimulus-response relation of sensory neurons in order to capture the observed signal processing operations and test them on responses to complex or natural stimuli [6-15].

A fundamental ingredient for such models is typically the receptive field, which describes the region of stimuli that affects a neuron's response. For retinal ganglion cells, the output neurons of the retina, spatial receptive fields are commonly used to capture how the cells respond to light spots of different sizes or to spatially structured visual stimuli, often by assuming that the cells linearly integrate signals over their receptive fields. Yet, responses under contrast-reversing spatial gratings have long revealed that many ganglion cells can display nonlinearities in their spatial signal integration [16-21], and it is thought that these spatial nonlinearities are also important under natural stimulation [8,9,22-26], in particular in view of sharp light intensity boundaries resulting from objects in visual scenes.

The source of these spatial nonlinearities appears to be the presynaptic bipolar cells $[10,27-$ 29], which provide the excitatory input to the ganglion cells and whose neurotransmitter release - despite the graded, non-spike-dependent synaptic exocytosis at their terminals can show partial rectification with respect to light intensity $[28,30]$. Yet, incorporating the nonlinear bipolar cell input into computational models has been difficult because determining the layout of bipolar cells and the nature of their nonlinearities either requires detailed anatomical knowledge [28] or data-intensive inference methods [10,12,31-35]. 
We therefore here seek a simple model that phenomenologically incorporates effects of nonlinear spatial stimulus integration and whose parameters can be obtained with relatively small amounts of data. The approach is based on identifying the receptive field of a ganglion cell and evaluating not only the (weighted) mean of light intensity across the receptive field, but also the variability of light intensity over space, measured by the (weighted) second-order statistics of the stimulus. Evaluated on recordings from salamander ganglion cells under flashed natural images, we find that this analysis reveals nonlinear spatial stimulus integration and provides a simple way to improve standard receptive-field-based models of ganglion cell activity.

\section{Results}

\section{Ganglion cell responses under stimulation with natural images}

To investigate responses of retinal ganglion cells to flashed natural images, we projected photographic images onto isolated salamander retinas and recorded the spiking activity of ganglion cells with multielectrode arrays. The images were presented individually in a pseudo-random sequence for $200 \mathrm{~ms}$ each, with an inter-stimulus-interval of $800 \mathrm{~ms}$ (Fig. 1A). Figure $1 \mathrm{~B}$ shows an example of one of the images, overlaid with the receptive field outline of a sample ganglion cell, and the spike patterns of this cell measured for 13 individual presentations of the image. A simple measure of the response is given by the total spike count elicited by the image presentation. To obtain the spike count, we used a temporal window ranging from image onset to $100 \mathrm{~ms}$ after image offset. Given the response latency of around $100 \mathrm{~ms}$ in these neurons [33], this window typically includes all spikes elicited by the image presentation, but excludes potential spikes that might be elicited by the withdrawal of the image.

Based on this spike count response measure, we found that stimulation with different images generated diverse, yet reliable response patterns: for a given ganglion cell, the range of spike counts typically varied between zero and around 10 to 15 , showing that a wide range of response strengths is elicited. For a given image, on the other hand, spike counts were highly reliable, with typical standard deviations of the spike count over repeated presentations of around one spike. Figure $1 \mathrm{C}$ shows the spike count responses to all 300 images for the sample ganglion cell. For each image, the variance of spike count over repeated trials was typically small and below the variability of a Poisson process (represented by the gray solid line), similar to findings of previous studies with non-natural stimuli $[36,37]$.

This reliability was observed for most cells. Figure 1D shows for each analyzed cell the average spike count variance (averaged over all images) as a measure of trial-by-trial noise in comparison to the average spike count over all images and trials as a measure of the average response strength. As for the sample cell in Figure 1C, the spike count variance was on average much smaller than the average spike count for most cell, indicating sub-Poissonian 
noise and high response reliability for individual images. Furthermore, the noise level was generally much smaller than the signal range covered by different images, as shown in Figure 1E. The trial-by-trial variability (again measured as the single-image spike-count variance averaged over images, $y$-axis) was for nearly all cells much below the signal range, as measured by the variance over images of the mean spike counts ( $x$-axis).

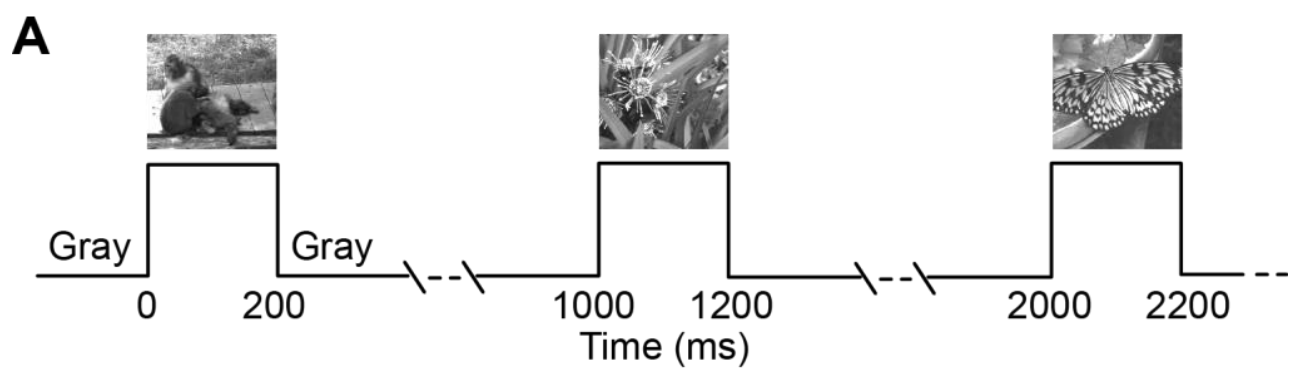

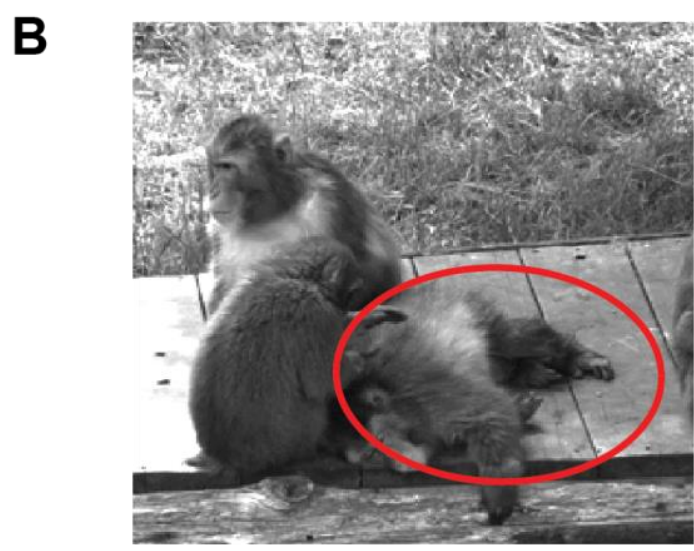
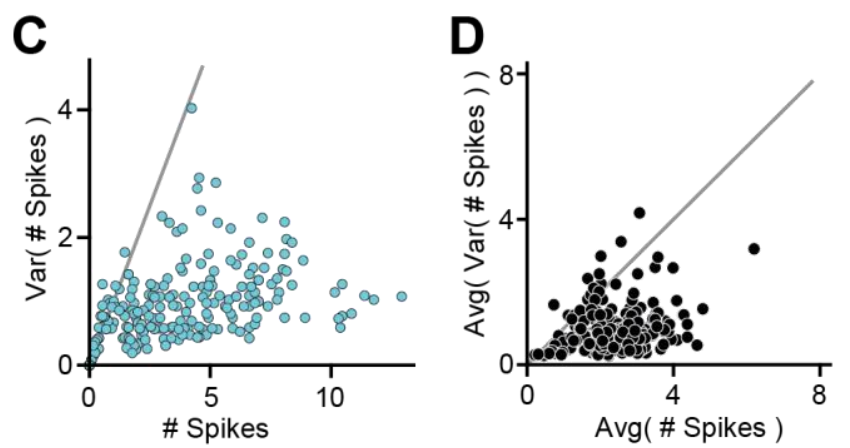
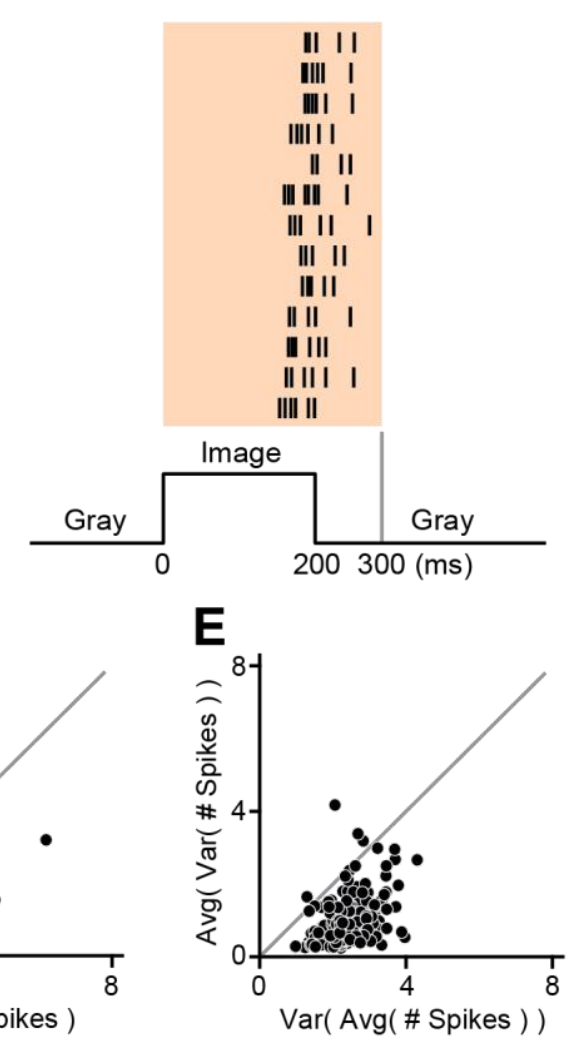

Figure 1. Overview of ganglion cell responses. A) Schematic of the sequence of 300 natural images presented individually in a pseudo-random fashion for $200 \mathrm{~ms}$ each, with an inter-stimulus-interval of $800 \mathrm{~ms}$. B) Left: One of the 300 natural images, overlaid with the 3-sigma outline of the receptive field of a sample retinal ganglion cell. Right: Raster plot of spike times recorded from the sample cell for 13 repeated presentations of this image. Every row corresponds to one image presentation. At the bottom, the time line of stimulus presentation is shown, indicating the 200 -ms presentation time, surrounded by periods of gray illumination. The vertical gray line at $300 \mathrm{~ms}$ marks the end of the applied window for counting spike numbers. $C$ ) Spike count variance ( $y$-axis) vs. average spike count ( $x$-axis) from the sample cell for each of the 300 images. The gray straight line denotes the expected relation of variance and average spike count for a Poisson process. D) Average over images of the single-image spike count variance as a measure of response noise ( $y$-axis) vs. the average spike count over all images as a measure of typical signal size (x-axis), shown for each recorded cell. E) Average over images of the single-image spike count variance as a measure of response noise ( $y$-axis) vs. variance over images of the average spike count per image as a measure of response range ( $x$-axis), shown for each recorded cell. 


\section{Local spatial contrast shapes ganglion cell responses}

To assess the relation of a cell's response to the presented natural image, we tested how the cell's spike count depended on simple image statistics inside the cell's receptive field. To do so, we determined the receptive field of a cell by standard reverse correlation [38]. The retina was visually stimulated with spatiotemporal white noise to calculate the average stimulus sequence that preceded a spike ("spike-triggered average"). Then, singular value decomposition was used to separate the spike-triggered average into a spatial and a temporal component. Finally, a two-dimensional Gaussian function was fitted to the spatial receptive field component to determine the center, size, and shape of the receptive field.

For a given image, we obtained the local stimulus for a cell by weighting the image with the 2D Gaussian representation of the receptive field. We then first considered the mean stimulus intensity $\left(I_{\text {mean }}\right)$ inside the receptive field by computing the mean pixel intensity of the local stimulus, corresponding to a linear integration of the stimulus across the receptive field. Figure 2A displays the relation between $I_{\text {mean }}$ and the measured spike count for a sample cell, which can be fitted by a parameterized nonlinear function (see Materials and Methods). Together, the linear image filtering by the receptive field and the nonlinear function constitute a classical linear-nonlinear (LN) model. To assess model performance, we used the coefficient of determination $R^{2}$ between the spike count data and the model's prediction for the images in a test set of 150 held-out images, which were not used for the fit of the nonlinear function. For the displayed sample cell, we found $R^{2}=0.65$, showing that $I_{\text {mean }}$ alone already had considerable predictive power, but did not completely specify the measured spike count.

We then asked whether - beyond mean stimulus intensity in the receptive field - spatial contrast contributed to determining the spike count. To do so, we assessed for each image the local spatial contrast (LSC) inside the receptive field by computing the standard deviation of pixel values in the local stimulus that had been obtained by weighting the image with the Gaussian representation of the receptive field. It is important to note, though, that the LSC is not independent of the mean stimulus intensity $I_{\text {mean. }}$. As depicted in Figure $2 \mathrm{~B}$, the LSC tended to be larger when $I_{\text {mean }}$ deviated more strongly from zero; both large positive and large negative deviations from mean light level favored a larger range of pixel intensities, as should be expected. Thus, a direction comparison of the LSC with the measured spike count, as shown for the sample cell in Figure $2 \mathrm{C}$, is not suited to determine whether the LSC itself affects the cell's response. High spike counts for large LSC values and positive correlations between LSC and spike count might have simply resulted from higher $I_{\text {mean }}$ values for the corresponding images.

To test more directly for how the LSC affected the spiking response, we aimed at assessing whether it influenced the response beyond the effect of $I_{\text {mean. }}$. To do so, we analyzed pairs of images that, for a given cell, yielded approximately equal $I_{\text {mean }}$ values and then computed the 
differences in spike count and in LSC for such image pairs [26]. Concretely, we ordered all images for a given cell according to their $I_{\text {mean }}$ value and compared spike count and LSC by calculating the differences $\Delta$ Spikes and $\triangle \mathrm{LSC}$ for each pair of neighbors in this ordered sequence. To verify that the residual differences in $I_{\text {mean }}$ did not have a major influence in this analysis, we also calculated their difference values $\Delta I_{\text {mean }}$ for the image pairs.

A

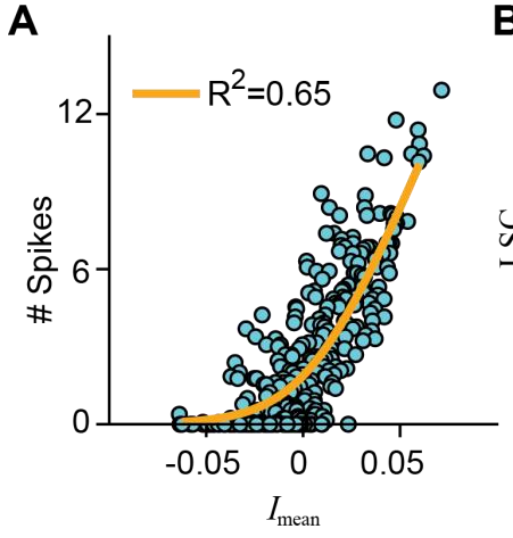

D

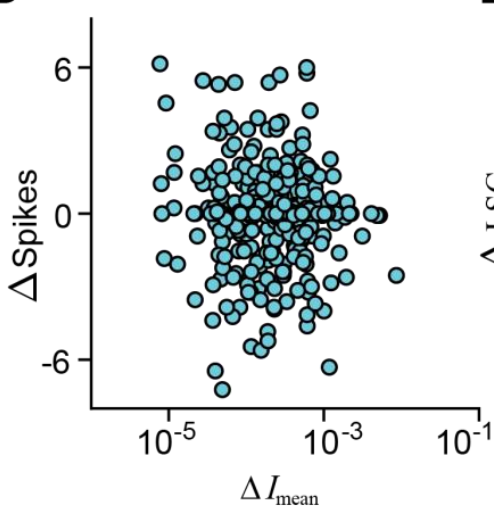

B

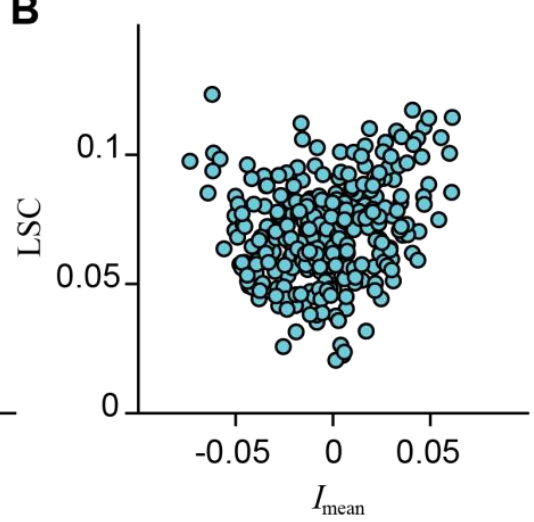

E

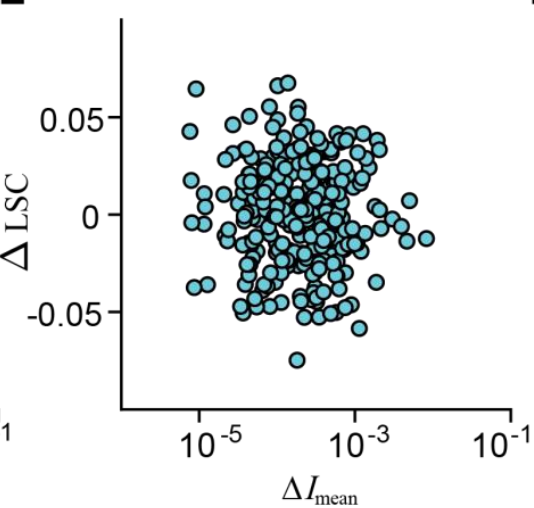

C

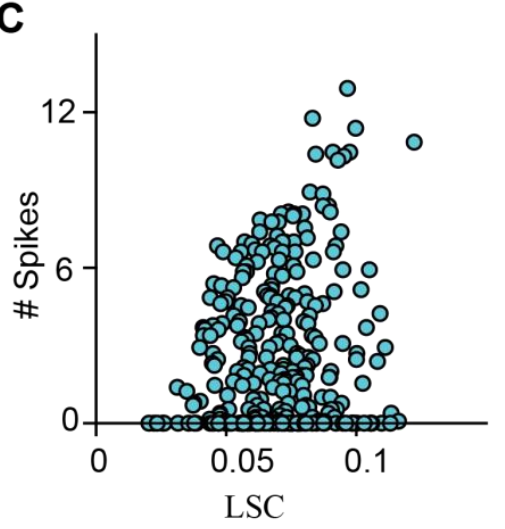

$\mathbf{F}$

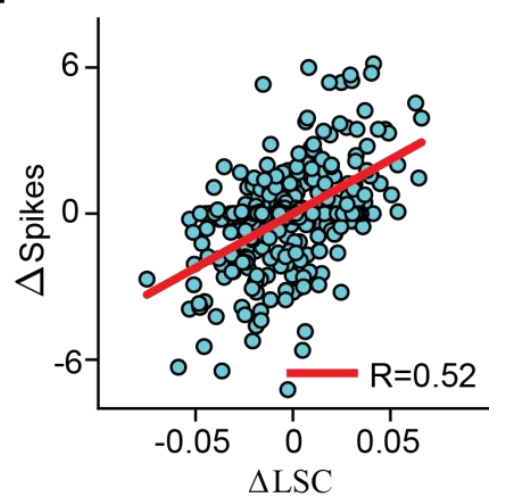

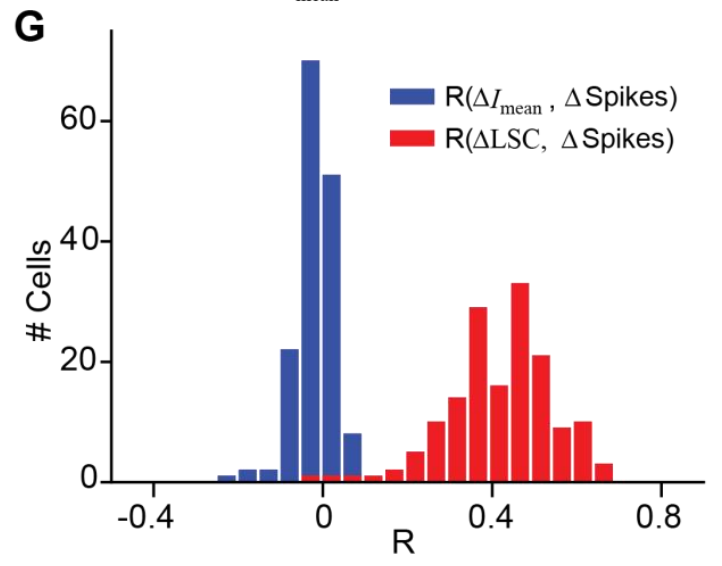

Figure 2. Effect of local spatial contrast on ganglion cell responses. A) Scatter plot of spike count vs. $I_{\text {mean }}$ for all 300 images for the sample cell of Figure 1B-C. The orange line shows the fitted nonlinearity cross-validated using 150 images, with the stated $R^{2}$ value used as an evaluation of the quality of the fit. B) LSC vs. I $I_{\text {mean }}$ for all images for the same sample cell. C) Spike count vs. LSC for all images for the same sample cell. D) Differences in $I_{\text {mean }}\left(\Delta I_{\text {mean }}\right)$ vs. differences in spike count ( $\Delta$ Spikes) for pairs of images that have neighboring values in the $I_{\text {mean }}$, plotted for the sample cell. E) As D, but for differences in local spatial contrast ( $\Delta \mathrm{LSC})$ vs. $\Delta I_{\text {mean }}$. F) Same as $\mathrm{D}$ and $\mathrm{E}$, but for $\Delta$ Spikes vs. $\Delta \mathrm{LSC}$. The red line is obtained by regression analysis, and the Pearson correlation coefficient is denoted in the plot.

G) Distributions over all recorded cells of the correlation coefficients $R$ for $\Delta I_{\text {mean }}$ vs. $\Delta$ Spikes (blue) and for $\triangle$ LSC vs. $\triangle$ Spikes (red). 
The scatter plots of $\Delta I_{\text {mean }}$ vs. $\Delta$ Spikes (Fig. 2D) and $\Delta I_{\text {mean }}$ Vs. $\Delta$ LSC (Fig. 2E) confirmed that the effects of mean stimulus intensity for the sample cell were abolished in this analysis. The residual $I_{\text {mean }}$ signal had no detectable influence on the spike count differences $\Delta$ Spikes and was not correlated with $\triangle \mathrm{LSC}$. In the absence of the influence of $I_{\text {mean }}$, we then found that the local spatial contrast systematically affected the spike count: $\Delta$ LSC and $\Delta$ Spikes displayed a pronounced correlation with $R=0.52$ (Fig. 2F). Population analysis corroborated the findings for the sample cell. When pairing images with similar mean stimulus inside a cell's receptive field, the spatial contrast differences $\triangle \mathrm{LSC}$ (but not the residual mean intensity differences $\Delta I_{\text {mean }}$ ) were generally positively correlated to the spike count differences $\Delta$ Spikes (Fig. $2 \mathrm{G}$ ), supporting that spatial contrast in the image can boost spike count beyond the effect of mean stimulus intensity.

\section{Spatial contrast model to incorporate sensitivity to spatial structure}

Next, we analyzed whether this additional information contained in the LSC about the spike count can improve the response prediction over the classical LN model. To do so, we set up a spatial contrast (SC) model, which combines information from the mean intensity and from the spatial contrast inside a cell's receptive field. The processing chain is explained in Figure 3A. The receptive field is found from responses to spatiotemporal flicker and fitted by a 2D Gaussian. The Gaussian provides weights for each image pixel to extract the local stimulus, whose distribution of pixel contrast values yields the local mean intensity $I_{\text {mean }}$ as the mean of this distribution and the local spatial contrast LSC as the standard deviation. The model's linear activation is computed as a weighted sum of the $I_{\text {mean }}$ and LSC values (Fig. 3B, left). Like in the classical LN model, the activation is turned into a prediction of the spike count through a nonlinear rectifying function. This function is fitted to the relation of the weighted sum of $I_{\text {mean }}$ and LSC and the measured number of spikes (Fig. 3B, center). The weight factor that is multiplied to the LSC is an additional free parameter and is fitted together with the parameters of the nonlinearity on the training data. The model is evaluated by computing the squared correlation coefficient $R^{2}$ on held-out images.

Figure $3 B$ (center) shows the relation between the activation of the SC model, as obtained from $I_{\text {mean }}$ and LSC together, and the spike counts of the test data for the sample cell of Figure 2. The nonlinear function captured the measured spike counts more accurately than in the classical LN model (cf. Fig. 2A). Including the spatial contrast information improved the model performance from $R^{2}=0.65$ for the classical $L N$ model to $R^{2}=0.76$. 
A
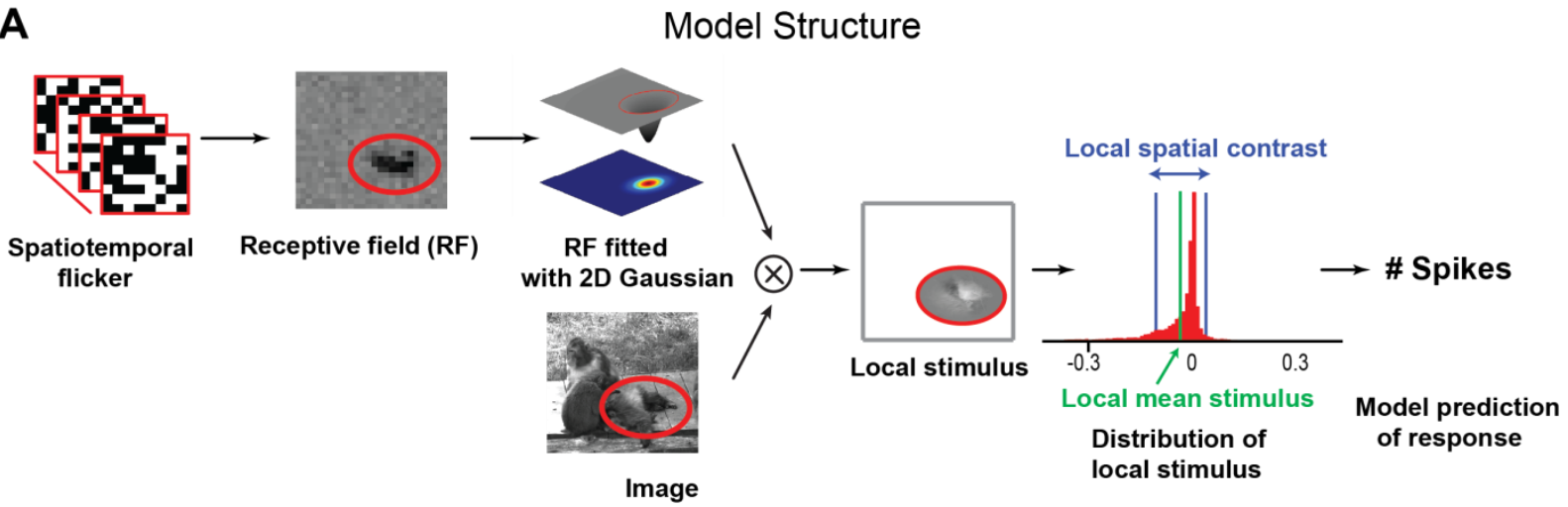

B
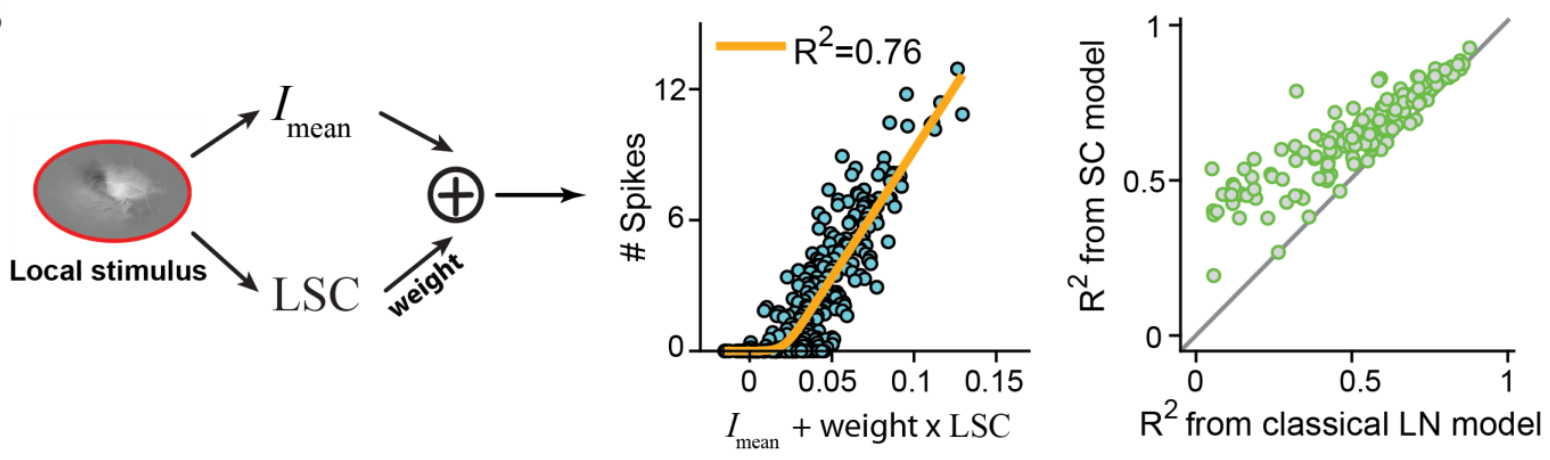

C
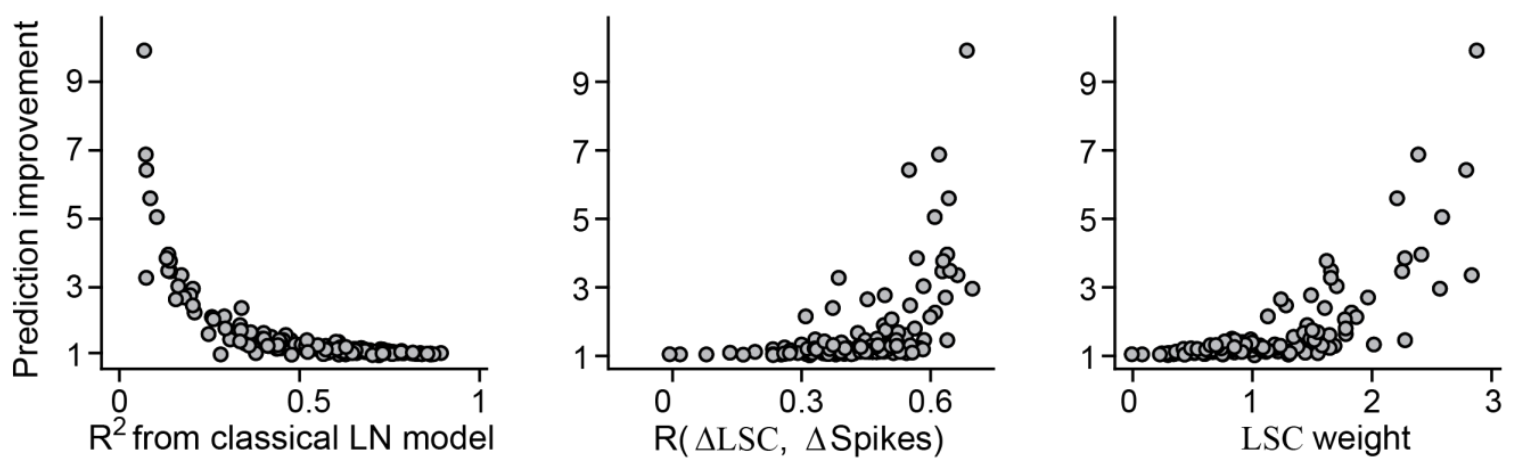

Figure 3. Modeling responses to natural images based on simple image statistics. A) Model for assessing the effect of simple image statistics on the spike count. Stimulation with spatiotemporal flicker is used to calculate a ganglion cell's spatial receptive field, which is then fitted by a 2D Gaussian function. Each natural image is cropped to the 3- $\sigma$ contour of the receptive field (red line) and weighted by the 2D Gaussian, yielding the local stimulus. The mean stimulus intensity $\left(I_{\text {mean }}\right)$ and the local spatial contrast (LSC) are obtained as the mean and the standard deviation of the pixel intensities in this local stimulus. Both measures are used in the prediction of the spike count response. B) Left: Schematic depiction of the SC model, which uses a weighted sum of the extracted values of $I_{\text {mean }}$ and the LSC for a given image. Center: Relationship between the linear signal of the SC model and the number of spikes recorded from the sample cell of Figure 2 for all images. The orange line shows the fitted nonlinearity, with the stated $R^{2}$ value used as an evaluation of the quality of the fit. Right: Coefficients of determination $R^{2}$ compared for the classical LN model, which takes $I_{\text {mean }}$ as input, and the SC model, which takes into account both $I_{\text {mean }}$ and LSC. C) Relative prediction improvement of the SC model over the classical LN model vs. model performance of the classical LN model (left), vs. the correlation coefficients calculated from $\triangle \mathrm{LSC}$ and $\triangle$ Spikes (center), and vs. the optimal LSC weights found in the fit of the SC model.

Population analysis corroborated the improved model performance of the SC model. The SC model had overall considerably better performance for predicting spike counts than the classical LN model (Fig. 3B, right), in particular when the classical LN model originally had low performance. This is also emphasized by plotting the prediction improvement, calculated as the ratio between $R^{2}$ from SC model and $R^{2}$ from the classical LN model, against the 
performance of the classical LN model (Fig. 3C, left). Furthermore, the improvement was larger when the analysis of spatial-contrast effects on spike count beyond the mean stimulus intensity (cf. Fig. 2F) revealed a sizeable correlation between $\triangle \mathrm{LSC}$ and $\Delta$ Spikes (Fig. $3 \mathrm{C}$, center). And the importance of the spatial contrast information for this improvement is also reflected in accompanying higher weights for the SC component in the model fits (Fig. 3C, right). These results suggest that spatial contrast inside the receptive field can exert a strong effect on spike responses beyond the mean stimulus intensity, and including this information can yield strongly superior models when the classical LN model fails.

\section{Assessing the relevant spatial scale of local spatial contrast}

We found that the LSC can be a useful predictor for ganglion cell spiking responses. So far, we have calculated this measure based on the standard deviation of image intensities at the level of individual pixels. This takes into account intensity variations at all spatial frequencies. Yet, it should be expected that spatial pooling by photoreceptors and bipolar cells prevents nonlinear stimulus integration for high spatial frequencies. In other words, intensity values of image points that are close together should be integrated linearly by the ganglion cell without any effect of how the total intensity is distributed between these image points. Thus, there should be an optimal spatial scale for calculating the LSC, and this optimal spatial scale should approximately correspond to the size of the relevant nonlinear subunits inside the receptive field, which are thought to correspond to bipolar cell receptive fields $[10,24,27,28]$.

To test for the optimal spatial scale, we varied the way in which we measured the LSC and searched for the highest predictive power of the SC model. Specifically, we looked at how spatial smoothing of the image before computing the LSC affected the response prediction. We expect that image predictions improve as long as smoothing occurs on spatial scales smaller than the subunits inside the receptive field, whereas smoothing at larger scales should degrade the predictions. The reason for this is that the right level of smoothing allows contrast variations on scales below the subfields to be averaged out (as we expect occurs inside the subunits), whereas contrast variations that span more than a single subunit do contribute to spike count prediction and their smoothing should deteriorate model performance. 
A

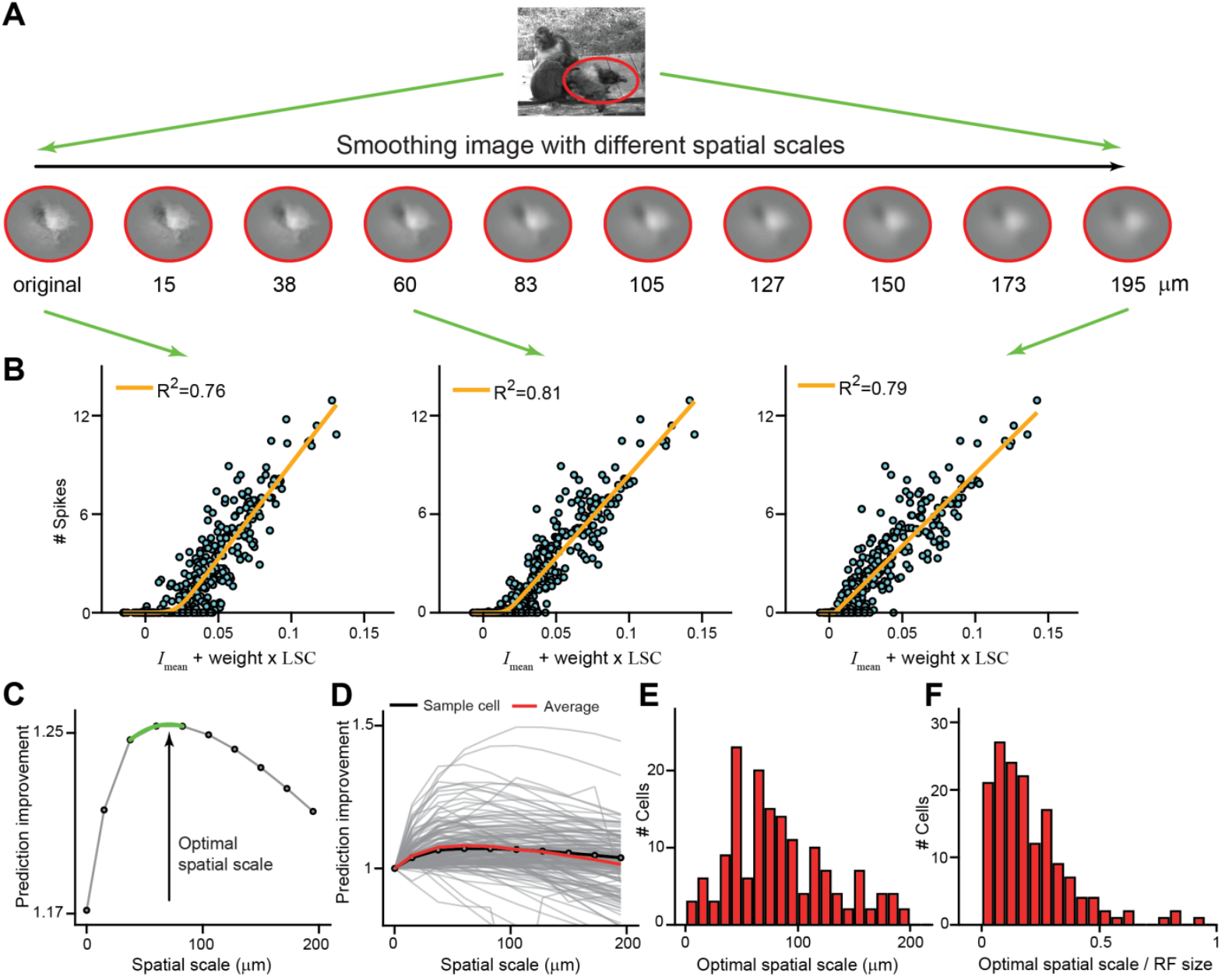

Figure 4. Analysis of the optimal spatial scale for calculating the local spatial contrast. A) Sample image overlaid with the 3- $\sigma$ outline of a sample cell's receptive field (red curve). Below: Local stimuli after smoothing with a 2D Gaussian filter with increasing spatial scale from 15 to $195 \mu \mathrm{m}$ and pixel-wise weighting by the sample cell's spatial receptive field. The first image is without smoothing. B) Relation between linear signal of the SC model and measured spike count, using the same $I_{\text {mean }}$ values, but LSC values derived from the different smoothed images, shown here for the original image and for the images with spatial scales of smoothing of 60 and $195 \mu \mathrm{m}$, respectively. The orange lines show the fitted nonlinearities and the $R^{2}$ values denote the corresponding model performance. C) Prediction improvement as a function of the level of smoothing for the sample cell. The optimal spatial scale is defined as the spatial scale at which $R^{2}$ reaches its maximum (as determined by the 2 nd-order polynomial fit around the maximal data point; green line). D) Prediction improvement as a function of the level of smoothing for all cells (gray curves), with the sample cell of $C$ shown as a black line. The red line displays an average over all cells. E) Distribution of optimal spatial scales. F) Distribution of optimal scales, normalized by each cell's receptive field size.

Figure 4A illustrates this analysis. The sample image on top, overlaid with a cell's receptive-field outline, was blurred by circular Gaussian filters with increasing spatial scale, ranging from 15 to $195 \mu \mathrm{m}$, and then pixel-wise multiplied by the cell's receptive field to yield the image patches shown below. In this way, we extracted for each image the LSC from the smoothed versions and combined this with the $I_{\text {mean }}$ from the original images to fit an SC model for each level of smoothing (Fig. 4B). As before, we assessed the model quality by the $R^{2}$ values and evaluated the model improvement (relative to the performance of the classical LN model) as a function of the spatial scale of smoothing (Fig. 4C). The spatial scale is here defined as three times the standard deviation of the Gaussian, for direct comparability with 
our definition of receptive field size. The optimal spatial scale is obtained as the spatial scale of smoothing for which $R^{2}$ is maximal, using interpolation with a second-order polynomial fitted to the three data points around the maximum data point (green line in Fig. 4C).

We performed this analysis for each cell (Fig. 4D) and extracted the optimal spatial scales. The distribution (Fig. 4E) shows a broad maximum in the range of 50-150 $\mu \mathrm{m}$. This is consistent with the typical size of bipolar cell receptive fields in the salamander retina $[39,40]$. When the optimal scale was normalized by the ganglion cell's receptive field size, the distribution (Fig. 4F) became somewhat narrower, with most ratios lying around or below a value of 0.3 . This suggests that subunits are typically smaller than a third of most ganglion cell's receptive field, consistent with about 10 or more subunits contributing to the receptive field.

\section{Discussion}

Current models of stimulus encoding by retinal ganglion cells often start with using a cell's receptive field as a spatial filter applied to incoming images. This is the case for the commonly applied LN model and for many of its extensions such as the generalized linear model $[41,42]$ or other approaches for including spike-timing dynamics or feedback loops $[43,44]$. The single-spatial-filter approach remains popular because it is conceptually straightforward, amenable to simple parameter-fitting approaches [38,41,42], and remarkably successful in capturing ganglion cell responses under specific stimulus conditions or for certain subtypes of ganglion cells $[6,9,26,41]$.

Yet, an underlying assumption of the simple linear spatial filtering is that all relevant nonlinearities of the system can be subsumed into processing at the output stage, following after stimulus integration over space (and time) has already taken place. This clashes with the wide-spread finding of nonlinear spatial integration, as revealed, for example, by reversing spatial gratings [16-20]. It is thought that this nonlinear spatial integration also affects responses of retinal ganglion cells to natural stimuli, leading to failures of the model approach with a single spatial filter $[7-10,12,26,45]$.

As the nonlinear spatial integration follows from the ganglion cell's nonlinear pooling of presynaptic bipolar cells with smaller receptive fields, the most principled way of constructing improved models has been to explicitly include this network structure by using multiple spatial filters - the model's subunits - in parallel and nonlinearly transforming the subunit signals before summation. This corresponds to a sequence of two LN models and is thus often referred to as an LNLN model. Yet, despite recent progress in inferring the layout and structure of the subunits $[10,12,28,34,35,46-48]$, obtaining such models remains challenging, and current procedures typically require large amounts of data.

In this work, we therefore take a different approach by assessing nonlinear spatial integration 
through a simple phenomenological model that goes beyond the standard spatial filtering approach by considering the local spatial contrast inside the receptive field, which we defined via the variability of stimulus intensities. We found that this spatial contrast was positively correlated to the spike count response of the recorded cells when effects of mean stimulus intensity were removed (Fig. 2). This analysis can serve as a simple and straightforward test for whether nonlinear spatial integration affects spike counts under natural stimuli. The measured direct effect of spatial contrast on spike count leads to a simple extension of the classical LN model approach by including spatial contrast as an additional model input. This yielded substantial improvements in predicting responses to natural images, in particular for cells where the performance of the classical LN model was poor (Fig. 3). Computing the spatial contrast measure after smoothing the image can further improve the model predictions and serve to test different spatial scales of nonlinear spatial integration (Fig. 4).

The spatial contrast model is phenomenological and does not provide much information about the layout of subunits or the nonlinearities that act on the subunit output. The strength lies in its simplicity, requiring only measurements of the receptive field and adding just a single free parameter as compared to the classical LN model. Thus, the model may serve to assess and partly capture effects of nonlinear spatial integration when little data is available. It may also be valuable for providing a simple benchmark for comparison with more complex models of nonlinear spatial integration when the classical LN model appears too simplistic for providing a baseline measure.

Structurally, the proposed model is similar to a generalized quadratic model, which allows for a general quadratic function of the stimulus and a subsequent nonlinear transformation [4951]. For the present case of natural image encoding, the stimulus is given as the set of pixel intensity values, and the general quadratic function could therefore incorporate the mean intensity via a linear component as well as the pixel standard deviation via a quadratic contribution. The difference of our approach is that we here use a particular, simple quadratic function of pixel contrast values, which does not require extensive parameter fitting, and that we use the standard deviation of pixel contrast values and thus supply the quadratic term with a square root. This provides an equal scaling of contributions from mean stimulus intensity and spatial contrast with overall stimulus contrast, which may be helpful considering the wide range of contrast values encountered in natural stimuli.

The simplicity of the spatial contrast model and its structural similarity to the LN model also make it amenable to different extensions, such as incorporating temporal filtering of the mean light intensity and the spatial contrast signal (either with identical or with potentially different filter shapes) and to temporal feedback, such as gain control signals or post-spike filters $[43,52]$. Moreover, it might serve as a useful, spatially nonlinear front end in more complex cascades of cortical visual processing [53-55]. Conversely, it may help include additional, often neglected nonlinear effects in models of the retina itself. This could be used, for example, 
to capture nonlinear spatial integration in the receptive field surround [56], for which subunit models have been difficult to set up, or nonlinear chromatic integration [57]. Similarly, using spatial contrast directly as an additional input channel could help include nonlinear effects in the outer retina, in the transmission from photoreceptors to bipolar cells [58-62]. Such a nonlinear front end could then be combined with the typical subunit model structure that is used to capture downstream nonlinear spatial integration in the connection from bipolar to ganglion cells at the inner retina [10,12,17,18,22,24,28,35,63-65].

\section{Acknowledgments}

This work was supported by the Deutsche Forschungsgemeinschaft (DFG, German Research Foundation) - project IDs 154113120 (SFB 889, project C01); 432680300 (SFB 1456, project B05) - by the European Union Seventh Framework Programme (FP7-ICT-2011.9.11) under grant agreement number 600954 (“VISUALISE”), and by the European Research Council (ERC) under the European Union's Horizon 2020 research and innovation programme (grant agreement number 724822). 


\section{Materials and Methods}

Electrophysiology. We used retinas from adult axolotl salamanders (Ambystoma mexicanum; pigmented wild type) of either sex. All experimental procedures were performed in accordance with national and institutional guidelines and were approved by the institutional animal care committee of the University Medical Center Göttingen (protocol number T11/35). Multielectrode array (MEA) recordings of ganglion cell spiking activity were obtained as described previously [66]. In brief, after dark-adaptation of the animal and enucleation of the eyes, retinas were peeled out of the eyecup and cut in half. One retina half was placed ganglion-cell-side-down on a planar multielectrode array (Multichannel Systems, 252 channels, 10- $\mu \mathrm{m}$ electrode diameter, 60- $\mu \mathrm{m}$ spacing) and perfused with oxygenated Ringer's solution (110 mM NaCl, $2.5 \mathrm{mM} \mathrm{KCl}, 1.6 \mathrm{mM} \mathrm{MgCl}$, $1.0 \mathrm{mM} \mathrm{CaCl}$, $22 \mathrm{mM} \mathrm{NaHCO}$, $10 \mathrm{mM}$ D-glucose, equilibrated with 95\% $\mathrm{O} 2$ and $5 \% \mathrm{CO} 2$ ). Recordings were performed at room temperature $\left(20^{\circ} \mathrm{C}-22^{\circ} \mathrm{C}\right)$. Potential spikes were detected by threshold crossing from the amplified voltage signals after band-pass filtering $(300 \mathrm{~Hz}$ to $5 \mathrm{kHz})$ and digitization at $10 \mathrm{kHz}$. Spike sorting was performed with a Gaussian mixture model [67]. Only well-separated units with a clear refractory period were used for further analysis.

Visual stimulation. Visual stimuli were projected onto the retina from a gamma-corrected miniature OLED monitor (eMagin, OLED-XL series, 800x600 pixels with a refresh rate of $60 \mathrm{~Hz}$ ). The monitor image was focused onto the photoreceptor layer via a telecentric lens to a pixel size of $7.5 \mu \mathrm{m} \times 7.5 \mu \mathrm{m}$. Stimuli were generated with a custom-made software, based on Visual $\mathrm{C}_{++}$and OpenGL. All stimuli had a mean light level of $I_{\text {mean }}=2.5 \mathrm{~mW} / \mathrm{m}^{2}$, which was also used as a background light level presented between stimuli.

Receptive fields were obtained from measurements with spatiotemporal white noise on a checkerboard layout with squares of $30 \mu \mathrm{m} \times 30 \mu \mathrm{m}$. For each square, light intensities were chosen randomly at a rate of $30 \mathrm{~Hz}$ from a binary distribution (100\% Michelson contrast). From the recorded spikes, we computed the spike-triggered average (STA) for each recorded ganglion cell [38], taking into account stimulus sequences of $660 \mathrm{~ms}$ before each spike. We used singular-value decomposition $[68,69]$ to decompose the STA into a temporal filter and a spatial receptive field and normalized each to unit Euclidean norm. Finally, we fitted a two-dimensional Gaussian function $G(\boldsymbol{x})=A \frac{1}{2 \pi \sqrt{|\Sigma|}} e^{-\frac{1}{2}(\boldsymbol{x}-\boldsymbol{\mu})^{T} \Sigma^{-1}(\boldsymbol{x}-\boldsymbol{\mu})}+B$ to the spatial receptive field, where $\boldsymbol{x}=(x, y)$ denotes the position in the image pixel space. The fit was obtained by least-squares optimization of the amplitude $A$, the receptive-field center position $\boldsymbol{\mu}$, the covariance matrix $\Sigma$, and the offset $B$. For further analysis (see "Models and response predictions" below), $G(\boldsymbol{x})$ was normalized by setting $A=1$ and $B=0$. The effective diameter of the receptive field was determined as $d=\sqrt{a \cdot b}$, where $a$ and $b$ are the major and minor axes of the 1.5-sigma contour of the fitted Gaussian. 
To stimulate the retina with natural images, we selected a set of 300 natural photographs from the McGill Calibrated Colour Image Database [70], displaying a wide range of natural and artificial scenes and all consistent with spanning a field of view of around $20-40^{\circ}$. Each image had a spatial resolution of $256 \times 256$ pixels, covering a total area of $1920 \mu \mathrm{m} \times 1920 \mu \mathrm{m}$ on the retina. The images were converted into grayscale by a weighted average of the RGB-color channels, using a ratio of $R: G: B=30: 59: 11$. Subsequently, all pixel values were shifted and scaled so that the mean pixel intensity of each image was equal to the background and the standard deviation was $50 \%$ of the mean intensity. Pixel values were clipped at $0 \%$ and $100 \%$ of the mean intensity to ensure compatibility with the light intensity range of the display. For each of the selected images, this occurred for fewer than $0.1 \%$ of the pixels. For all analyses, stimuli are represented by the Weber contrast $C$ at each pixel, $C=\left(L-L_{\text {mean }}\right) / L_{\text {mean }}$, where $L$ is the pixel light level and $L_{\text {mean }}$ is the average light level over the image.

Images were presented individually for $200 \mathrm{~ms}$ each in a pseudo-random sequence, separated by $800 \mathrm{~ms}$ of background illumination. Responses of individual ganglion cells were quantified as the number of spikes over a $300-\mathrm{ms}$ window following stimulus onset. Given the response latency of around $100 \mathrm{~ms}$, this generally excludes spikes elicited by the disappearance of the image after $200 \mathrm{~ms}$.

Models and response predictions. To assess the relevance of spatial structure in natural images for shaping ganglion cell responses, we compared two models for predicting spike counts. The first model is a classical Linear-Nonlinear (LN) model, which takes the cell's receptive field as a spatial filter that is applied to the stimulus. The model thus integrates light intensity signals linearly over a ganglion cell's receptive field. The second model, which we call Spatial Contrast (SC) Model, has a similar structure as the classical LN Model, but takes an additional, second input besides the linearly filtered light intensity. This second input is a measure of spatial contrast inside the receptive field, which is obtained from the standard deviation of the (weighted) pixel intensities.

Concretely, both models start with filter signals $F_{\mathrm{LN}}$ and $F_{\mathrm{SC}}$, respectively. For a given image, $F_{\mathrm{LN}}$ was the mean stimulus intensity $I_{\text {mean }}$, given by the average Weber contrast as seen through the cell's receptive field. This was obtained by filtering the image with the receptive-field fit $G(\boldsymbol{x})$ :

$$
F_{\mathrm{LN}}=I_{\text {mean }}=\frac{1}{N} \sum_{i=1}^{N} G\left(\boldsymbol{x}_{i}\right) \cdot C\left(\boldsymbol{x}_{i}\right)
$$

where $i$ enumerates all pixel locations $\boldsymbol{x}_{i}$ within the 3-sigma contour of $G(\boldsymbol{x}), N$ is the number of these pixels, and $C\left(\boldsymbol{x}_{i}\right)$ is the corresponding pixel contrast.

$F_{\mathrm{SC}}$, on the other hand, received an additional input, given by the local spatial contrast (LSC), which was computed as the standard deviation of the pixel intensities: 


$$
\text { LSC }=\sqrt{\frac{1}{N-1} \sum_{i=1}^{N}\left(G\left(\boldsymbol{x}_{i}\right) \cdot C\left(\boldsymbol{x}_{i}\right)-I_{\text {mean }}\right)^{2}}
$$

where $i$ and $N$ are defined as above. Note that this is the pixel standard deviation of the filtered image; alternatively, the local spatial contrast could be computed as the weighted standard deviation of the original image [26], but the difference between these measures is small.

The obtained measure of local spatial contrast was added to the filtered image signal with a weight $w$ as a free parameter:

$$
F_{\mathrm{SC}}=I_{\text {mean }}+w \cdot \mathrm{LSC}
$$

To turn $F_{\mathrm{LN}}$ and $F_{\mathrm{SC}}$ into predictions for natural images, we computed nonlinearities for both models from the natural images. This was done by relating $F_{\mathrm{LN}}$ and $F_{\mathrm{SC}}$ to the average evoked spike count in a histogram fashion: For each model, the filter signal $\left(F_{\mathrm{LN}}\right.$ or $\left.F_{\mathrm{SC}}\right)$ was binned into 40 bins with equal numbers of data points, and the average filter signal and average of corresponding spike counts were computed for each bin. The histograms were fitted with nonlinear "softplus" functions of the form $r\left(F_{\mathrm{X}}\right)=a_{1} \cdot \ln \left(1+e^{a_{2} \cdot\left(F_{\mathrm{X}}+a_{3}\right)}\right)$, where $F_{\mathrm{X}}$ stands for $F_{\mathrm{LN}}$ or $F_{\mathrm{SC}}$, by optimizing the parameters $a_{1}, a_{2}$, and $a_{3}$ (together with the weight $w$ in the case of the SC model) according to a least-squares criterion. The fitted functions were obtained with 150 natural images and used to obtain response predictions for another 150 held-out natural images. To quantify model performance, we computed for each model the correlation coefficient $R$ between prediction and measured firing rate and reported the explained variance $R^{2}$.

We recorded 9 retinas to collect 215 cells. Using the spike numbers $N_{\mathrm{sp}}(B)$ and $N_{\mathrm{sp}}(W)$ in response to full-field black and white stimuli $( \pm 100 \%$ contrast), respectively, we classified cells into 169 OFF cells with $N_{\mathrm{sp}}(B) / N_{\mathrm{sp}}(W)>3,9$ ON cells with $N_{\mathrm{sp}}(W) / N_{\mathrm{sp}}(B)>3$, and 37 ON-OFF cells otherwise. For consistency, we only selected OFF cells for further analyses and excluded cells if the maximum average response to any of the 300 natural images was smaller than 5 spikes, leaving us with 156 cells for the final analysis.

Data availability. The spike-train data recorded for this work have been made available at https://gin.g-node.org/gollischlab/Liu Gollisch 2021 RGC spiketrains spatial contrast mod el [71]. 


\section{References}

1. Rust NC, Movshon JA. In praise of artifice. Nat Neurosci. 2005;8: 1647-1650. doi:10.1038/nn1606

2. Felsen G, Dan Y. A natural approach to studying vision. Nat Neurosci. 2005;8: 16431646. doi:10.1038/nn1608

3. Carandini M, Demb JB, Mante V, Tolhurst DJ, Dan Y, Olshausen BA, et al. Do we know what the early visual system does? J Neurosci. 2005;25: 10577-10597. doi:10.1523/JNEUROSCI.3726-05.2005

4. Simoncelli EP, Olshausen BA. Natural image statistics and neural representation. Annu Rev Neurosci. 2001;24: 1193-1216. doi:10.1146/annurev.neuro.24.1.1193

5. Rieke F, Rudd ME. The challenges natural images pose for visual adaptation. Neuron. 2009;64: 605-616. doi:10.1016/j.neuron.2009.11.028

6. Nirenberg S, Pandarinath $\mathrm{C}$. Retinal prosthetic strategy with the capacity to restore normal vision. Proc Natl Acad Sci U S A. 2012;109: 15012-15017. doi:10.1073/pnas.1207035109

7. Freeman J, Field GD, Li PH, Greschner M, Gunning DE, Mathieson K, et al. Mapping nonlinear receptive field structure in primate retina at single cone resolution. Elife. 2015;4: e05241. doi:10.7554/eLife.05241

8. Heitman A, Brackbill N, Greschner M, Sher A, Litke AM, Chichilnisky EJ. Testing pseudo-linear models of responses to natural scenes in primate retina. bioRxiv. doi:10.1101/045336

9. Turner $\mathrm{MH}$, Rieke F. Synaptic rectification controls nonlinear spatial integration of natural visual inputs. Neuron. 2016;90: 1257-1271. doi:10.1016/j.neuron.2016.05.006

10. Liu JK, Schreyer HM, Onken A, Rozenblit F, Khani MH, Krishnamoorthy V, et al. Inference of neuronal functional circuitry with spike-triggered non-negative matrix factorization. Nat Commun. 2017;8: 149. doi:10.1038/s41467-017-00156-9

11. Walker EY, Sinz FH, Cobos E, Muhammad T, Froudarakis E, Fahey PG, et al. Inception loops discover what excites neurons most using deep predictive models. Nat Neurosci. 2019;22: 2060-2065. doi:10.1038/s41593-019-0517-x

12. Shah NP, Brackbill N, Rhoades C, Kling A, Goetz G, Litke AM, et al. Inference of nonlinear receptive field subunits with spike-triggered clustering. Elife. 2020;9. doi:10.7554/eLife.45743 
13. Coen-Cagli R, Kohn A, Schwartz O. Flexible gating of contextual influences in natural vision. Nat Neurosci. 2015;18: 1648-1655. doi:10.1038/nn.4128

14. Lesica NA, Jin J, Weng C, Yeh C-I, Butts DA, Stanley GB, et al. Adaptation to stimulus contrast and correlations during natural visual stimulation. Neuron. 2007;55: 479-491. doi:10.1016/j.neuron.2007.07.013

15. Rowekamp RJ, Sharpee TO. Cross-orientation suppression in visual area V2. Nat Commun. 2017;8: 15739. doi:10.1038/ncomms15739

16. Enroth-Cugell C, Robson JG. The contrast sensitivity of retinal ganglion cells of the cat. J Physiol. 1966;187: 517-552. doi:10.1113/jphysiol.1966.sp008107

17. Hochstein S, Shapley RM. Linear and nonlinear spatial subunits in $Y$ cat retinal ganglion cells. J Physiol. 1976;262: 265-284. doi:10.1113/jphysiol.1976.sp011595

18. Demb JB, Haarsma L, Freed MA, Sterling P. Functional circuitry of the retinal ganglion cell's nonlinear receptive field. J Neurosci. 1999;19: 9756-9767. doi:10.1523/JNEUROSCI.19-22-09756.1999

19. Petrusca D, Grivich MI, Sher A, Field GD, Gauthier JL, Greschner M, et al. Identification and characterization of a Y-like primate retinal ganglion cell type. J Neurosci. 2007;27: 11019-11027. doi:10.1523/JNEUROSCI.2836-07.2007

20. Krieger B, Qiao M, Rousso DL, Sanes JR, Meister M. Four alpha ganglion cell types in mouse retina: Function, structure, and molecular signatures. PLOS ONE. 2017;12: e0180091. doi:10.1371/journal.pone.0180091

21. Crook JD, Packer OS, Dacey DM. A synaptic signature for ON- and OFF-center parasol ganglion cells of the primate retina. Vis Neurosci. 2014;31: 57-84. doi:10.1017/S0952523813000461

22. Schwartz G, Rieke F. Perspectives on: information and coding in mammalian sensory physiology: nonlinear spatial encoding by retinal ganglion cells: when $1+1 \neq 2$. J Gen Physiol. 2011;138: 283-290. doi:10.1085/jgp.201110629

23. Cao X, Merwine DK, Grzywacz NM. Dependence of the retinal ganglion cell's responses on local textures of natural scenes. J Vis. 2011;11: 11. doi:10.1167/11.6.11

24. Gollisch T. Features and functions of nonlinear spatial integration by retinal ganglion cells. J Physiol Paris. 2013;107: 338-348. doi:10.1016/j.jphysparis.2012.12.001

25. Turner MH, Sanchez Giraldo LG, Schwartz O, Rieke F. Stimulus- and goal-oriented frameworks for understanding natural vision. Nat Neurosci. 2019;22: 15-24. 
doi:10.1038/s41593-018-0284-0

26. Karamanlis D, Gollisch T. Nonlinear spatial integration underlies the diversity of retinal ganglion cell responses to natural images. J Neurosci. 2021;41: 3479-3498. doi:10.1523/JNEUROSCI.3075-20.2021

27. Demb JB, Zaghloul K, Haarsma L, Sterling P. Bipolar cells contribute to nonlinear spatial summation in the brisk-transient $(\mathrm{Y})$ ganglion cell in mammalian retina. J Neurosci. 2001;21: 7447-7454. doi:10.1523/JNEUROSCI.21-19-07447.2001

28. Schwartz GW, Okawa H, Dunn FA, Morgan JL, Kerschensteiner D, Wong RO, et al. The spatial structure of a nonlinear receptive field. Nat Neurosci. 2012;15: 1572-1580. doi:10.1038/nn.3225

29. Kuo SP, Schwartz GW, Rieke F. Nonlinear spatiotemporal integration by electrical and chemical synapses in the retina. Neuron. 2016;90: 320-332. doi:10.1016/j.neuron.2016.03.012

30. Borghuis BG, Marvin JS, Looger LL, Demb JB. Two-photon imaging of nonlinear glutamate release dynamics at bipolar cell synapses in the mouse retina. J Neurosci. 2013;33: 10972-10985. doi:10.1523/JNEUROSCI.1241-13.2013

31. Victor JD, Shapley RM. The nonlinear pathway of $Y$ ganglion cells in the cat retina. J Gen Physiol. 1979;74: 671-689. doi:10.1085/jgp.74.6.671

32. Victor JD. The dynamics of the cat retinal Y cell subunit. J Physiol. 1988;405: 289-320. doi:10.1113/jphysiol.1988.sp017334

33. Bölinger D, Gollisch T. Closed-loop measurements of iso-response stimuli reveal dynamic nonlinear stimulus integration in the retina. Neuron. 2012;73: 333-346. doi:10.1016/j.neuron.2011.10.039

34. Shi Q, Gupta P, Boukhvalova AK, Singer JH, Butts DA. Functional characterization of retinal ganglion cells using tailored nonlinear modeling. Sci Rep. 2019;9: 8713. doi:10.1038/s41598-019-45048-8

35. Maheswaranathan N, Kastner DB, Baccus SA, Ganguli S. Inferring hidden structure in multilayered neural circuits. PLOS Comput Biol. 2018;14: e1006291. doi:10.1371/journal.pcbi.1006291

36. Berry MJ, Warland DK, Meister M. The structure and precision of retinal spike trains. Proc Natl Acad Sci U S A. 1997;94: 5411-5416. doi:10.1073/pnas.94.10.5411

37. Berry MJ, Meister M. Refractoriness and neural precision. J Neurosci. 1998;18: 2200- 
2211. doi:10.1523/JNEUROSCI.18-06-02200.1998

38. Chichilnisky EJ. A simple white noise analysis of neuronal light responses. Network. 2001;12: 199-213.

39. Wu SM, Gao F, Maple BR. Functional architecture of synapses in the inner retina: segregation of visual signals by stratification of bipolar cell axon terminals. J Neurosci. 2000;20: 4462-4470. doi:10.1523/JNEUROSCI.20-12-04462.2000

40. Baccus SA, Ölveczky BP, Manu M, Meister M. A retinal circuit that computes object motion. J Neurosci. 2008;28: 6807-6817. doi:10.1523/JNEUROSCI.4206-07.2008

41. Pillow JW, Shlens J, Paninski L, Sher A, Litke AM, Chichilnisky EJ, et al. Spatio-temporal correlations and visual signalling in a complete neuronal population. Nature. 2008;454: 995-999. doi:10.1038/nature07140

42. Paninski L. Maximum likelihood estimation of cascade point-process neural encoding models. Network. 2004;15: 243-262.

43. Berry MJ, Brivanlou IH, Jordan TA, Meister M. Anticipation of moving stimuli by the retina. Nature. 1999;398: 334-338. doi:10.1038/18678

44. Keat J, Reinagel P, Reid RC, Meister M. Predicting every spike: a model for the responses of visual neurons. Neuron. 2001;30: 803-817. doi:10.1016/s0896-6273(01)00322-1

45. Mclntosh LT, Maheswaranathan N, Nayebi A, Ganguli S, Baccus SA. Deep Learning Models of the Retinal Response to Natural Scenes. Adv Neural Inf Process Syst. 2016;29: 1369-1377.

46. Vintch B, Movshon JA, Simoncelli EP. A convolutional subunit model for neuronal responses in macaque V1. J Neurosci. 2015;35: 14829-14841. doi:10.1523/JNEUROSCI.2815-13.2015

47. Real E, Asari H, Gollisch T, Meister M. Neural circuit inference from function to structure. Curr Biol. 2017;27: 189-198. doi:10.1016/j.cub.2016.11.040

48. Deny S, Ferrari U, Macé E, Yger P, Caplette R, Picaud S, et al. Multiplexed computations in retinal ganglion cells of a single type. Nat Commun. 2017;8: 1964. doi:10.1038/s41467-017-02159-y

49. Park IM, Pillow JW. Bayesian spike-triggered covariance. Adv Neural Inf Process Syst. 2011;24: 1692-1700. 
50. Park IM, Archer E, Priebe N, Pillow JW. Spectral methods for neural characterization using generalized quadratic models. Adv Neural Inf Process Syst. 2013;26: 2454-2462.

51. Rajan K, Marre O, Tkačik G. Learning quadratic receptive fields from neural responses to natural stimuli. Neural Comput. 2013;25: 1661-1692. doi:10.1162/NECO_a_00463

52. Pillow JW, Paninski L, Uzzell VJ, Simoncelli EP, Chichilnisky EJ. Prediction and decoding of retinal ganglion cell responses with a probabilistic spiking model. J Neurosci. 2005;25: 11003-11013. doi:10.1523/JNEUROSCI.3305-05.2005

53. Lindsey J, Ocko SA, Ganguli S, Deny S. A unified theory of early visual representations from retina to cortex through anatomically constrained deep CNNs. 7th International Conference on Learning Representation (ICLR). New Orleans, LA: OpenReview.net; 2019. Available: https://openreview.net/forum?id=S1xq3oR5tQ

54. Eliasmith C, Stewart TC, Choo X, Bekolay T, DeWolf T, Tang Y, et al. A large-scale model of the functioning brain. Science. 2012;338: 1202-1205. doi:10.1126/science.1225266

55. Bradley C, Abrams J, Geisler WS. Retina-V1 model of detectability across the visual field. J Vis. 2014;14: 22-22. doi:10.1167/14.12.22

56. Takeshita D, Gollisch T. Nonlinear spatial integration in the receptive field surround of retinal ganglion cells. J Neurosci. 2014;34: 7548-7561. doi:10.1523/JNEUROSCI.0413-14.2014

57. Khani MH, Gollisch T. Linear and nonlinear chromatic integration in the mouse retina. Nat Commun. 2021;12: 1900. doi:10.1038/s41467-021-22042-1

58. Schreyer HM, Gollisch T. Nonlinear spatial integration in retinal bipolar cells shapes the encoding of artificial and natural stimuli. Neuron. 2021;109: 1692-1706. doi:10.1016/j.neuron.2021.03.015

59. Baden T, Schubert T, Chang L, Wei T, Zaichuk M, Wissinger B, et al. A tale of two retinal domains: near-optimal sampling of achromatic contrasts in natural scenes through asymmetric photoreceptor distribution. Neuron. 2013;80: 1206-1217. doi:10.1016/j.neuron.2013.09.030

60. Clark DA, Benichou R, Meister M, Azeredo da Silveira R. Dynamical adaptation in photoreceptors. PLOS Comput Biol. 2013;9: e1003289. doi:10.1371/journal.pcbi.1003289

61. Endeman D, Kamermans M. Cones perform a non-linear transformation on natural stimuli. J Physiol. 2010;588: 435-446. doi:10.1113/jphysiol.2009.179036 
62. Howlett MHC, Smith RG, Kamermans M. A novel mechanism of cone photoreceptor adaptation. PLOS Biol. 2017;15: e2001210. doi:10.1371/journal.pbio.2001210

63. Enroth-Cugell C, Freeman AW. The receptive-field spatial structure of cat retinal $\mathrm{Y}$ cells. J Physiol. 1987;384: 49-79. doi:10.1113/jphysiol.1987.sp016443

64. Crook JD, Peterson BB, Packer OS, Robinson FR, Troy JB, Dacey DM. Y-cell receptive field and collicular projection of parasol ganglion cells in macaque monkey retina. $\mathrm{J}$ Neurosci. 2008;28: 11277-11291. doi:10.1523/JNEUROSCI.2982-08.2008

65. Wienbar S, Schwartz GW. The dynamic receptive fields of retinal ganglion cells. Prog Retin Eye Res. 2018;67: 102-117. doi:10.1016/j.preteyeres.2018.06.003

66. Liu JK, Gollisch T. Spike-Triggered Covariance Analysis Reveals Phenomenological Diversity of Contrast Adaptation in the Retina. PLOS Comput Biol. 2015;11: e1004425. doi:10.1371/journal.pcbi.1004425

67. Pouzat $\mathrm{C}$, Mazor O, Laurent G. Using noise signature to optimize spike-sorting and to assess neuronal classification quality. J Neurosci Methods. 2002;122: 43-57. doi:10.1016/s0165-0270(02)00276-5

68. Wolfe J, Palmer LA. Temporal diversity in the lateral geniculate nucleus of cat. Vis Neurosci. 1998;15: 653-675. doi:10.1017/s0952523898154068

69. Gauthier JL, Field GD, Sher A, Greschner M, Shlens J, Litke AM, et al. Receptive fields in primate retina are coordinated to sample visual space more uniformly. PLOS Biol. 2009;7: e1000063. doi:10.1371/journal.pbio.1000063

70. Olmos A, Kingdom FAA. A biologically inspired algorithm for the recovery of shading and reflectance images. Perception. 2004;33: 1463-1473. doi:10.1068/p5321

71. Liu JK, Gollisch T. Dataset - Salamander retinal ganglion cell responses to natural images. G-Node. 2021. doi:10.12751/g-node.kod28e 